\title{
From an object-superiority effect to an object-inferiority effect with movement of the fixation point
}

\author{
BRUCE EARHARD and ROSEANNE ARMITAGE \\ Dalhousie University, Halifax, Nova Scotia B3H 4JI, Canada
}

\begin{abstract}
The object-superiority effect (OSE) refers to the fact that target lines can be more accurately discerned when part of a cube-like, three-dimensional object context than when part of a flat, unconnected line context (Weisstein \& Harris, 1974). Explanations of the OSE have been based on the assumption that it is the configurational properties of the object form, such as its three dimensionality, and coherence that are responsible for the effect. It is demonstrated that the OSE produced by stimuli such as those used by Weisstein and Harris does not depend exclusively on configurational variables. Evidence is presented that fixation-point location and the amount of line detail about the point of fixation are crucial.
\end{abstract}

For a period of time it was believed that it might be possible to account for form perception in terms of a hierarchical, feature-analyzer system along lines suggested by Selfridge (1959). Physiological studies provided clear evidence of neurons in the visual system capable of responding to the presence of simple trigger features in the environment such as lines and edges in appropriate orientation, and there was reason to believe that these neurons were part of a larger, convergent-hierarchical, neural structure capable of cumulatively integrating lower order information about lines, edges, orientation, etc., into the more complex realities of conventional experience (Barlow, 1972; Hubel \& Wiesel, 1962, 1965). Such a "datadriven" or "bottom-up" view of form perception has not weathered well. Subsequent analyses have afforded little support for rigidly serial neural hierarchies (e.g., Sekular, 1974), and there is very strong behavioral evidence to suggest that "top-down" or "conceptually guided" behavior is important (Lindsay \& Norman, 1977). The strongest evidence of "top-down" processing is provided by context effects in speech and visual perception. In the area of visual perception, for example, Williams and Weisstein (1978) have demonstrated what they term an "object-line effect," that is, that line components can be detected more accurately when they are part of a coherent, three-dimensional, cube-like object form than when they are presented alone. If the higher order configurational character of the form is dependent upon the prior detection of lower order line elements, then it is difficult to see how,

This research was supported by grants from the Natural Sciences and Engineering Research Council of Canada. We are grateful to J. Barresi, R. Klein, P. Jusczyk, and M. Ozier for their encouragement and advice. Requests for reprints should be addressed to B. Earhard, Department of Psychology, Dalhousie University, Halifax, Nova Scotia B3H 4J1, Canada. in terms of a simple convergent hierarchical model, higher order configurational variables can influence discrimination of lower order line components. At the very least, some sort of feedback mechanism has to be incorporated into the system to allow higher order structures to influence the attention directed toward lower order components in the analytic process as, for example, in the model of Milner (1974).

It is obvious that context effects can play a very important role in shaping theory formation, and because of this, much interest has centered on specifying the mechanism underlying context effects. One approach has been to try to determine if provision of a context effectively serves to reduce an absolute judgment task to a relative judgment task and thereby facilitate anchoring a stimulus in space or, more generally, to reduce the uncertainty associated with responding. While appealingly simple, such an approach has received little experimental support. Schendel and Shaw (1976) did find that they could eliminate a context effect involving detection of two line elements by providing a subject with the two-alternative choices prior to presentation-a finding which suggests that the function of context may indeed be to reduce uncertainty and that the effect may be overridden by providing subjects with the possible alternatives prior to presentation. More recent experimentation has shown, however, that a pronounced context effect can be readily obtained in experiments involving a choice between two line elements in which alternatives are provided prior to presentation (Earhard, 1980; Williams \& Weisstein, 1978). As far as spatial anchoring is concerned, Williams and Weisstein (1978) took steps to largely eliminate the benefit context could provide with respect to anchoring, and a pronounced context effect remained evident (see also Pomerantz, Sager, \& Stoever, 1977).

What has emerged as the most likely underlying 
determinant of the context effect in perception is the configurational character of the context. Weisstein and her associates have emphasized, in particular, the importance of such global configurational variables as three dimensionality, connectedness, and coherence in considerations of the context effect (e.g., Weisstein \& Maguire, 1980; Williams \& Weisstein, Note 1). To support this perspective, they cite the well-established object-superiority effect (OSE), which shows that a target line can be discerned more readily if it is part of a coherent, connected, and three-dimensional object context than if it is part of a flat, unconnected line context (Weisstein \& Harris, 1974). They cite also the fact that, in the case of the object-line effect, the target line is discriminated more accurately within a context than when alone if it is contained within a connected, threedimensional object context, but not if it is contained within a flat, unconnected line context. Other investigators have illustrated the importance of Gestalt configurational factors as determinants of whether contexts facilitate or impair discrimination of component elements (e.g., Banks \& Prinzmetal, 1976; Pomerantz, Sager, \& Stoever, 1977; Prinzmetal \& Banks, 1977).

The present paper focuses on the object-superiority effect. It represents an attempt to determine whether an exclusively configurational interpretation of the OSE, generated with Weisstein and Harris' type of forms, is possible. Previous research on the objectline context effect with these forms suggests that context effectiveness depends crucially on the location and the amount of line detail of the fixation point. Earhard (1980) has shown (1) that Williams and Weisstein's (1978) finding that line elements can be more accurately discerned when part of a threedimensional context than when alone can be totally reversed by a small movement of the fixation point, and (2) that, if the fixation point is positioned at locations containing substantial amounts of visual detail, normally effective contexts can cease to be effective and even impair target-line discrimination. To obtain similar results in the case of the OSE would strengthen the contention that fixation-point location and the amount of visual detail at the fixation point are more important in the study of context effectiveness than has been assumed, and confirm that the OSE demonstrated with forms of the Weisstein and Harris type is not determined solely by configurational variations among the forms.

\section{EXPERIMENT 1}

Experiment 1 was a preliminary study that compared the best three-dimensional object context used by Weisstein and Harris (1974) in their demonstration of the object-superiority effect with their least

effective, flat, unconnected line context with respect to their capacity to facilitate discrimination of a constituent line element. The object of interest was to determine whether the well-established superiority of the object context would be evident, not only when the fixation point was located in the normal position shown in Figure 1a, which is free of line detail, but also when the fixation point rested at the point where there is a concentration of line detail as shown in Figure $2 a$. If our assumptions about the negative effects of line detail at and about the fixation point are correct, the shift in fixation point to the new location should very substantially reduce the effectiveness of the object context.

\section{Method}

Stimuli. The four different variants of the best and poorest of Weisstein and Harris' forms shown in Figures $1 \mathrm{a}$ and $1 \mathrm{~b}$ and Figures $2 \mathrm{a}$ and $2 \mathrm{~b}$, respectively, were selected for two reasons. First, these forms provided an opportunity to sample the capacity of subjects to discriminate target lines that fell along all four diagonals, and second, this assembly of forms provided an arrangement such that it would be possible in subsequent experiments to systematically alter the amount of visual detail on and

\section{SET A}

a

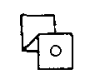

b

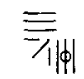

C

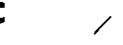

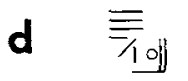

e
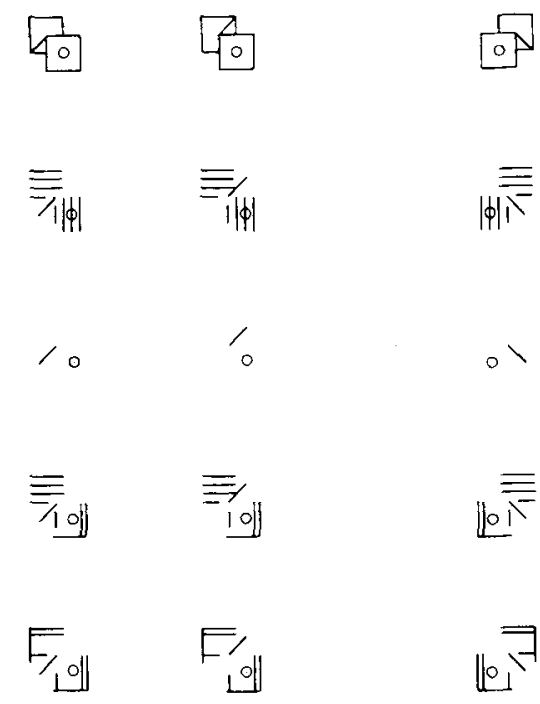

SET

B

\section{f}
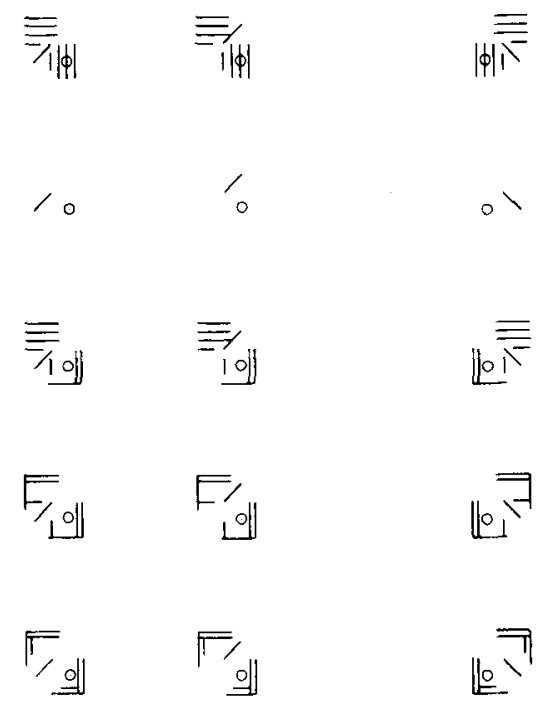

$\circ$

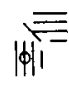

$\circ$

Figure 1. Rows 1a, 1b, and 1c show, respectively, the object context, regular line context, and target-line elements used in Part A of Experiment 1 and in all parts of Experiment 3. Rows 1d, le, and if show, respectively, the modifications of the regular line context employed in Parts $A, B$, and $C$ of Experiment 3. Closed circles were not part of the stimulus configurations; they are included only to clarify location of the fixation point, which was identical to that used by Weisstein and Harris (1974). 


\section{SET A}

a
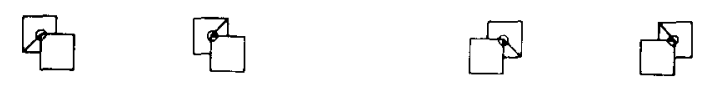

b
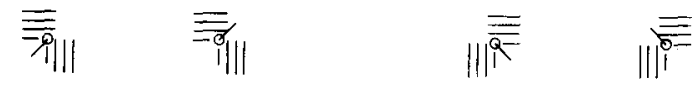

c
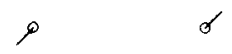

Q
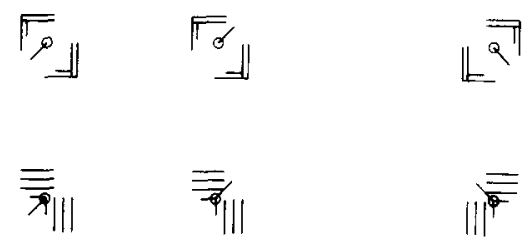

$\left.|1|\right|^{\phi}$

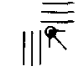

Figure 2. Rows $2 a, 2 b$, and $2 c$ show, respectively, the object context, the regular line context, and target-line elements with the fixation-point location used in Part $B$ of Experiment 1 and in Experiment 2. Rows $2 \mathrm{~d}$ and $2 \mathrm{e}$ show, respectively, the outline and altered line contexts used in Experiment 2. Closed circles were not part of stimulus configurations. They are included only to clarify location of the fixation point.

around the fixation point and target line. Stimuli were separated into Sets A and B for presentation purposes. This was necessary because, as inspection of Figures 1 and 2 will show, contexts in Set B are mirror images of contexts in Set A. To combine all four forms in a single presentation sequence would allow subjects the opportunity to use mirror-image differences in contexts as a basis for reducing uncertainty as to which of the four target lines had been presented. Accordingly, a given subject was presented with stimuli from Set A or Set B, but not both.

A Koh-i-nor Rapidomatic pen with a $.25-\mathrm{mm}$ nib was used to draw stimulus context on white bristol board. Target lines were $5.8 \mathrm{~mm}$ in length. The overall width of the object and line contexts shown in Figures 1 and 2 was $12 \mathrm{~mm}$. The height of the object context was $12 \mathrm{~mm}$, and, as in the Weisstein and Harris study, the line context was slightly higher at $14 \mathrm{~mm}$. At a viewing distance of $60 \mathrm{~cm}$, target lines subtended a visual angle of $.56 \mathrm{deg}$. The object and line contexts in overall width subtended a visual angle of $1.14 \mathrm{deg}$. In height, the object and line forms subtended visual angles of 1.14 and 1.34 deg, respectively.

Procedure. There were two parts to the experiment. In Part A, subjects were presented with the Weisstein and Harris forms with the conventional fixation-point location shown in Figures $1 \mathrm{a}$ and $\mathrm{lb}$; in Part $\mathrm{B}$, the same forms were presented with the new, more central, fixation-point location shown in Figures 2a and $2 \mathrm{~b}$. A Gerbrands two-field tachistoscope was used to present stimuli to the subjects. The subjects fixated on a faint pencil dot about $.35 \mathrm{~mm}$ in diameter and, when instructed to proceed by the experimenter, pressed a button which resulted in the removal of the preexposure field and a $20 \mathrm{-msec}$ presentation of the exposure field containing stimulus material (but no fixation point), followed by the immediate reappearance of the preexposure field. Before the start of the presentation sequence, subjects were presented with examples of contexts from either Set A or Set B. They were informed that the object of the experiment was for

them to detect, and report by pointing at a display card during an exposure, which of the two target line elements associated with a given type of context had been presented. The display card contained the two target line elements from Set A or Set B in appropriate location with respect to the fixation point as shown in Figures $1 \mathrm{c}$ and $2 \mathrm{c}$. The level of illumination of the exposure field was fixed at $3.38 \mathrm{~cd} / \mathrm{m}^{2}$. The level of illumination of the preexposure field was adjusted for every subject so as to ensure an overall performance level of $70 \%-80 \%$ correct responding. The mean level of illumination of the preexposure field was $6.83 \mathrm{~cd} / \mathrm{m}^{2}$ in Part A and $6.54 \mathrm{~cd} / \mathrm{m}^{2}$ in Part B.

Two blocks of 32 practice presentations were given to familiarize the subjects with the procedures and to adjust the level of illumination of the preexposure fields so as to ensure that the requisite level of correct responding was obtained. This was followed by 12 blocks of test presentations. Object and line contexts were presented in separate, alternating, 32-item presentation blocks. The order of items within a presentation block was random, with the restriction that no more than four presentations of the same target-line-context combination could occur in immediate succession. After each presentation block there was a pause long enough for the experimenter to tally the number of correct responses. Any adjustment to illumination level of the preexposure field was made, if necessary, only after both object and line contexts had been presented equally often. Twenty undergraduates, who received course credit for participating, served as subjects. All subjects were between 17 and 28 years of age, and all were naive with respect to the purpose of the study. There were four females and four males in Part $A$ and seven females and five males in Part B. Half the subjects in both parts of the study were presented with contexts from Set $A$, and the other half with contexts from Set $B$. The subjects were given no feedback about their performance levels.

\section{Results}

The typically observed superiority of the object context over the line context was evident in Part A. Target lines within the object context were identified correctly on $80.7 \%$ of the presentations, but within the line context on only $73.1 \%$ of the presentations. $A$ very different pattern was evident in Part $B$. The subjects detected target lines on $78.4 \%$ of the presentations in the case of the line context, but on only $73.4 \%$ of the presentations in the case of the object context. An overall analysis of variance performed on the number of correct responses confirmed that there was a very pronounced interaction, reflecting the different effect of context on performance in Parts $A$ and $B[F(1,18)=11.34, p<.01]$. There was no overall difference in accuracy level in the two parts of the experiment $[F(1,18)=1.45, p<.10]$. This was not unexpected, however, since a fixed level of performance was maintained by adjusting luminance levels of the preexposure fields.

The results of the study confirm the importance of fixation-point location. A relatively small movement of the fixation-point location effectively eliminated the object-superiority effect. The interesting question, of course, is how the simple movement of the point of fixation can eliminate the normal facilitating effect of a good context. Our contention, as indicated in the introduction, is that it is the amount of line detail 
located on and about the fixation point and the target line that is the crucial factor. Such a contention certainly conforms with the consequences of fixationpoint movement in the present study. Inspection of Figures 1a and $1 \mathrm{~b}$ will show that, in the object context, the fixation point rests on a central area free of visual detail but that in the line context it falls upon the middle of a vertical line, with other line elements interposed between the fixation point and the to-be-detected target line. The shift in fixation shown in Figures $2 a$ and $2 b$ reversed the imbalance of line detail located at the point of fixation. In this instance, it is in the object context that the fixation point falls upon the junction point of target and context lines; in the line context, fixation rests on only one end of the target line and adjacent lines do not intersect with the target line at the point of fixation. While the interpretation offered is compatible with the findings, it could be much strengthened if it could be shown that systematically altering the amount of line detail on or about the fixation point and target line radically changed the effectiveness of a given context. Experiment 2 was directed toward this objective.

\section{EXPERIMENT 2}

Experiment 2 had three specific objectives. The first was to determine whether the effectiveness of a context could be enhanced by removing line detail from on and around the fixation point and target line. The second was to determine whether the effectiveness of a context could be impaired by causing a small amount of line detail to converge upon the fixation point. The third was to provide a replication of the finding in Experiment 1 that the normally greater effectiveness of the object context reported by Weisstein and Harris (1974) can be eliminated by simply moving the fixation point to the location shown in Figure 2.

There were two parts to the study. The contexts used in Part A consisted of regular line and object contexts from Experiment 1 with the fixation point located at one end of the target line (Figures $2 a$ and $2 b)$ and an additional, outline, context as shown in Figure $2 \mathrm{~d}$. The additional form possessed the same number of line elements as the object and line contexts, but these line elements were moved as far as possible from the target line without doing fatal injury to the general outline of the context. If our assumptions were correct, the absence of line detail about the fixation point and the target line would make detection of target-line elements easiest in the outline context, even though it lacked three dimensionality and connectedness, followed by the regular line coutext and, finally, the object context, in which performance would be poorest, as in Experiment 1, because context and target lines converged at the fixation point.

The contexts used in Part B consisted of the object and outline contexts from Part A (Figures $2 a$ and $2 \mathrm{~d}$, respectively) and a slightly altered version of the regular line context shown in Figure 2e. Comparison of the altered line context with the regular line context shown in Figure $2 b$ will confirm that the only change in the altered version is that the two shortest line elements, usually removed $2 \mathrm{~mm}$ from the target line, were now joined with the target line. Given these three contexts, the expectation was again that the lack of line detail about the fixation point and the target line would make the outline context most effective in facilitating target-line detection, but that the alteration to the line context produced by joining target and context lines at the point of fixation would impair very substantially the effectiveness of the altered line context and make performance on the altered line context at least as poor as that evident in the object context.

\section{Method}

Stimuli. The object and line contexts were those used in Part B of Experiment 1. The new outline form shown in Figure $2 \mathrm{~d}$ was identical to the object and line forms in height, but was $1.5 \mathrm{~mm}$ greater in width, subtending a visual angle of $1.29 \mathrm{deg}$. As in Experiment 1, all forms were drawn on bristol board with a .25-mm nib.

Subjects and Procedure. Sixteen subjects were recruited. They were between the ages of 17 and 28 and were naive with respect to the objective of the experiment. There were four males and four females in Part $A$ and five males and three females in Part B. Except for one male in Part $A$ and one female in Part $B$, who were graduate volunteers, subjects were undergraduate volunteers, who received course credit points for participating. The procedures were, in the main, similar to those in Experiment 1. Half of the subjects in each part of the study were presented contexts from Set $\mathbf{A}$ and half with contexts from Set B. In both Part A and Part $B$, the three different contexts were presented in separate 32 -item presentation blocks. Within each presentation block, items were randomly arranged, with the restriction that no more than four presentations of the same target-line-context combination could occur in immediate succession within a block. A total of 18 different blocks of test presentations were given over three sessions. Each type of context was presented equally often. The order of presentation was random, except for the restriction that a new presentation block containing examples of a given context could not be presented until all contexts had been presented equally often. The same 20-msec-exposure duration used in Experiment 1 was employed in Experiment 2, and the subjects were required to indicate their responses after each presentation by pointing at a display with the two appropriate alternative target lines drawn from either Set A or Set B. Each session was preceded by three practice blocks to familiarize, or refamiliarize, the subjects with the procedures and to allow the level of illumination to be adjusted if necessary to ensure that an overall performance level of $70 \%-80 \%$ correct responding be maintained. The level of illumination of the exposure field was the same as in Experiment 1 , and the mean level of illumination of the preexposure field was $5.15 \mathrm{~cd} / \mathrm{m}^{2}$ in Part A and $4.85 \mathrm{~cd} / \mathrm{m}^{2}$ in Part B. If any adjustments in illumination level were required during the course of test presentations, they were carried out only after all contexts had been presented equally often. 


\section{Results}

Our first objective was to determine whether removing line detail from on and about the fixation point and target line would facilitate detection of the target line. As may be seen in Figure 3, the answer is that it does. The subjects were much more accurate in discerning a target line when it was contained within the outline context, where line detail had been moved from the fixation point and target line, than when it was contained in object or line context. The mean advantage of the outline context over the object context was $19.3 \%$ in Part A and $16.0 \%$ in Part B. This pattern was evident in the data of all subjects and reliable in both Part $\mathrm{A}[\mathrm{t}(7)=10.69, \mathrm{p}<.01]$ and Part $B[t(7)=7.72, p<.01]$. Similarly, comparisons of the outline context with the line context showed a mean advantage favoring the outline context over the regular line context of $8.0 \%$ in Part $\mathrm{A}$; over the altered line context in Part B (which was expected to impair performance), the advantage was even more pronounced-28.2\%. The advantage was evident in the data of all subjects in Part B and of all but one subject in Part A. Mean differences are significant in both Part $\mathrm{A}[\mathrm{t}(7)=4.02, \mathrm{p}<.01]$ and Part $B[t(7)=20.39, p<.01]$. The marked superiority of the outline context confirms that moving line detail from on and about the fixation point and target line can facilitate context effectiveness.

The remaining objectives were to determine (1) whether the finding of Experiment 1-that, with the fixation point location used, the normally greater effectiveness of the object context over the line context is eliminated-could be replicated, and (2) whether moving line detail so that it converged upon the fixation point, as in the altered line context of Part B, would impair context effectiveness. It can be seen in Figure 3 that both objectives were met. In Part A, as in Experiment 1, the subjects were more accurate in specifying the target line when it was contained within the regular line context than when it was in the object context, but in Part B, in which the two small line elements were moved so that they joined the target line and point of fixation, the direction of the difference was reversed, and the effectiveness of the altered line context was impaired to such a degree that it was inferior to the object context. This pattern was apparent in the data of all subjects. The $11.3 \%$ advantage of the regular line context over the object context was reliable in Part $\mathrm{A}[\mathrm{t}(7)=4.90, \mathrm{p}<.01]$, as was the very substantial $12.2 \%$ disadvantage of the altered line context vis à vis the object context in Part $B[t(7)=7.29, p<.01]$.

While we had expected that the altered line context would impair detection of target-line elements, we had not anticipated such a large effect and can only speculate about its cause. One possibility is that, in the case of the altered line context, there may be an

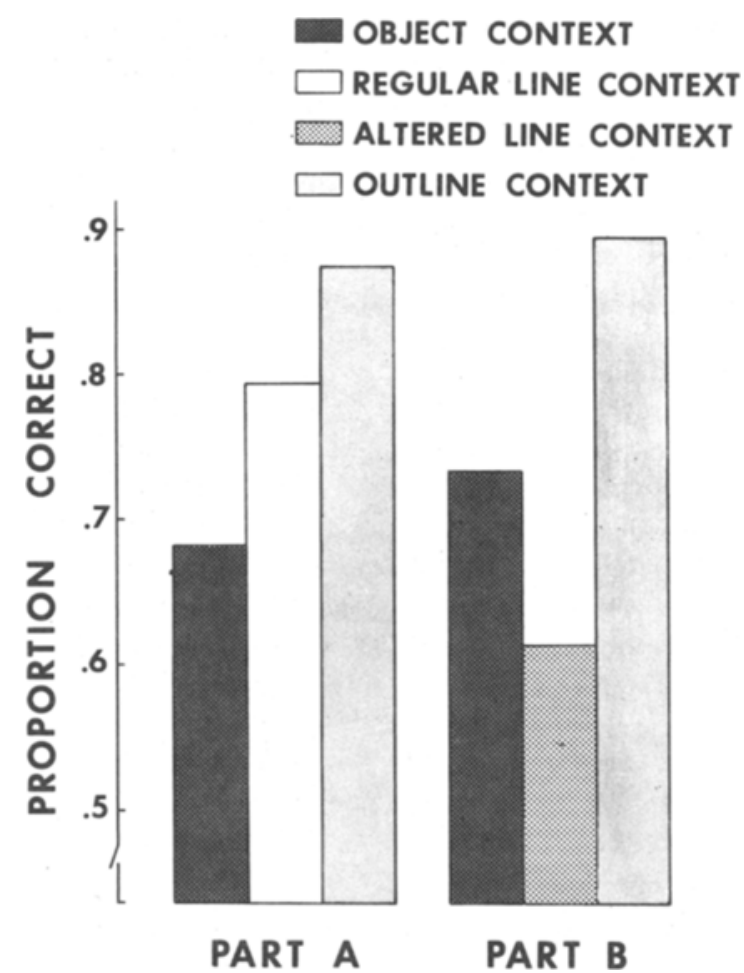

Figure 3. The mean proportion of correct target-line designations made by subjects in Parts $A$ and $B$ of Experiment 2. Contexts and fixation-point location are as shown in Figure 2.

additional detrimental interaction between the local structure produced by joining the two short context lines with the target line and nearby line elements that is absent in the object context. Some justification for such an interpretation is afforded by the results of the line manipulations undertaken in Experiment 3.

Experiment 2 thus confirms the finding of Experiment 1 that a shift in the fixation point can effectively eliminate the object superiority effect. It provides, in addition, considerable support for our contention that line detail on and about the fixation point can reduce the effectiveness with which a context can facilitate detection of target-line elements. Removing line detail from on and about the fixationpoint and target line greatly facilitated the effectiveness of a context, and moving line detail so that it converged upon the fixation point and target line profoundly impaired target-line detection.

\section{EXPERIMENT 3}

Experiment 3 sought to explore further the effects of fixation location and the amount of line detail on and about the fixation point. The Weisstein and Harris forms have been typically employed with the 
fixation point located in the position shown in Figures $1 \mathrm{a}$ and $1 \mathrm{~b}$. Under such circumstances, it has been repeatedly found that target lines are much more accurately detected when they are part of the object context than when they are part of the line context (e.g., Klein, 1978; McClelland, 1978; Womersley, 1977; this study, Experiment 1). The argument advanced earlier in the paper to explain the advantage of the object form over the regular line context was that, in the case of the object form, the fixation point rests on an area free of visual detail, whereas, in the case of the line context, not only does it rest on the middle of a vertical line, but also other lines are interposed between the fixation point and surround the target line. Given such an argument, we should be able to demonstrate that, with the regular fixation-point location (as shown in Figure 1, the systematic movements of line detail away from the fixation-point and target-line locations will eliminate the normally observed superiority of the three-dimensional object context over the flat, unconnected line context.

\section{Method}

Stimuli. The three different modified line contexts used are shown in Figures 1d, le, and 1f. In the first modification (Figuie 1d), line detail was moved, as shown, away from the fixation point location; in the second modification (Figure le), visual detail was removed from the upper part of the line context above the target line; and in the third modification (Figure 1f), the remaining short vertical and horizontal lines were moved away from the target line to produce the same outline context used in Experiment 2. The object and regular line contexts were identical to those used in the preceding studies. All modifications of the line form were the same height as the regular line and object forms but were, as in Experiment 2,1.5 mm wider and subtended a visual angle of $1.29 \mathrm{deg}$.

Subjects and Procedure. There were three parts to the study. In each part a different modified line context was compared with the object context and the regular line context shown in Figu:es $1 \mathrm{a}$ and $1 \mathrm{~b}$. In Part A, the modified line context shown in Figure 1d was compared with object and regular line contexts; in Part B, the comparison involved the modified line context shown in Figure le; and in Part C, it involved the modified context shown in Figure if. A different group of eight subjects served in each part of the experiment. All subjects were undergraduates, between the ages of 17 and 28, who received course credit for their participation. There were equal numbers of female and male subjects in Parts A and C and five female and three male subjects in Part B. All subjects were naive with respect to the objectives of the study. Half the subjects received contexts from Set $A$, and half received them from Set $B$. The number of presentations, presentation procedure, and exposure parameters in each of the three parts of the experiment were identical to those used in Experiment 2. As in the preceding studies, the level of illumination of the preexposure field was adjusted so as to ensure an overall performance level of between $70 \%$ and $80 \%$ correct responding. The mean level of illumination of the preexposure field was $5.65 \mathrm{~cd} / \mathrm{m}^{2}$ in Part A, $4.85 \mathrm{~cd} / \mathrm{m}^{2}$ in Part B, and $5.13 \mathrm{~cd} / \mathrm{m}^{2}$ in Part C.

\section{Results}

Figure 4 shows the proportion of correct targetline detection when target lines were part of the object context, the regular line context, and the dif-

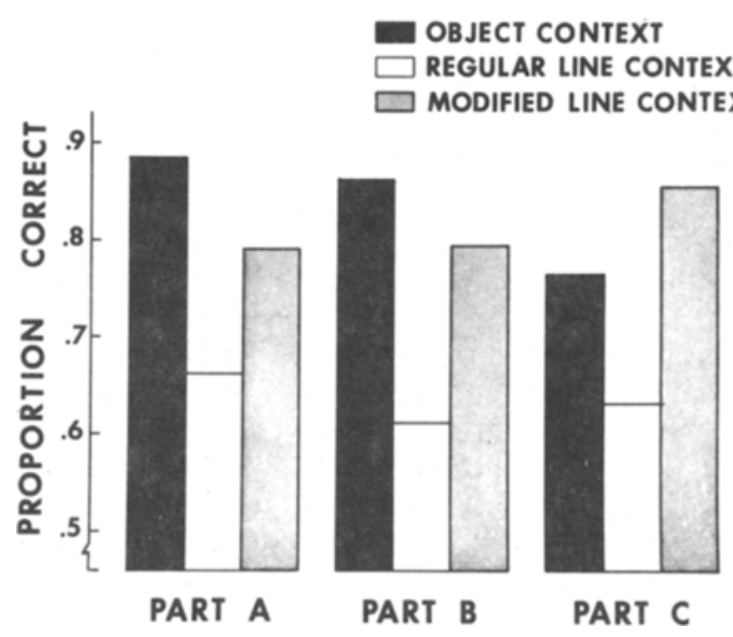

Figure 4. The mean proportion of correct target-line designations made by subjects in Parts $A, B$, and $C$ of Experiment 3 . Contexts and fixation-point location are as shown in Figure 1. The modified line contexts shown in Parts $A, B$, and $C$ represent performance on context modifications shown, respectively, in Rows 1d, 1e, and 1f of Figure 1.

ferent modified line contexts used in the three parts of the study. There are three observations worth making. First, the typically observed superiority of the object context over the regular line context was evident. Second, removal of even a small amount of visual detail from the fixation point area of the modified line context in Part $\mathrm{A}$ made it a more effective context than the regular line context. Third, and most important, the removal of a substantial amount of visual detail from the fixation-point and targetline areas in Part $\mathrm{C}$ rendered the modified (outline) line context superior to the object context, as in Experiment 2 .

The advantage of the object context over the regular line context was evident in the data of all individual subjects in all three parts of the study. The mean advantage of the object context over the regular line context was $22.4 \%$ in Part $\mathrm{A}, 25.4 \%$ in Part B, and $13.5 \%$ in Part C. Separate t tests showed the advantage of the object over the line context to be reliable in Part $\mathrm{A}[\mathrm{t}(7)=21.31, \mathrm{p}<.01]$, Part B $[\mathrm{t}(7)=18.88, \mathrm{p}<.01]$, and Part $\mathrm{C}[\mathrm{t}(7)=5.60$, $\mathrm{p}<.01]$. It is difficult to specify the reason for the reduction in magnitude of the difference between the object and line contexts in Part C. It may, in part, reflect individual differences. Indeed, in Part A of Experiment 1, the magnitude of the difference was only $7.6 \%$. A more likely contributing factor is, however, that the design of the study required performance to be maintained within the $70 \%-80 \%$ range of correct responding, that the increase in the effectiveness of the modified (outline) context in Part $\mathrm{C}$ had to be compensated for by a reduction in the level of performance of other contexts to main- 
tain this overall performance level, and that this, to some degree, depressed the range of allowable differences. Whatever the reason, a substantial and reliable difference remained evident.

The modified line contexts were found to be more effective than the regular line context by all subjects in all three parts of the study. The mean difference favoring the modified line context was $13.0 \%$ in Part A, $18.5 \%$ in Part B, and $22.6 \%$ in Part C. Separate $t$ tests indicated that differences in Part $\mathrm{A}[\mathrm{t}(7)$ $=12.25, \mathrm{p}<.01]$, Part $\mathrm{B}[\mathrm{t}(7)=13.31, \mathrm{p}<.01]$, and Part $\mathrm{C}[\mathrm{t}(7)=10.89, \mathrm{p}<.01]$ were reliable. The data confirm that moving line detail from the fixation point and the target line can greatly facilitate the effectiveness of a line context.

While the modified line contexts were consistently more effective than regular line contexts in all three parts of the study, the modifications introduced in Parts $A$ and $B$ were not sufficient to make either of the modified contexts involved more effective than the object context. In fact, all subjects were significantly more accurate when target lines were contained in the object context than when they were in the modified line context [Part $A, t(7)=8.89$, $\mathrm{p}<.01$; Part $\mathrm{B}, \mathrm{t}(7)=4.73, \mathrm{p}<.01]$. The mean advantage of the object context over the modified line context in Part A was 9.4\%; in Part B, it was $6.9 \%$. It was not until Part $C$, when all line detail was removed from on and about the fixation point and target line of the modified line context to produce the same outline context used in Experiment 2, that there was a substantial advantage in favor of the modified (outline) context over the object context. All subjects were more accurate in detecting the target line in the outline context, and the overall advantage of $9.1 \%$ was reliable $[\mathrm{t}(7)=12.72$, $p<.01]$. The results obtained in Part $C$ with the outline context are similar to those obtained in Experiment 2 , and they demonstrate again that, if the fixation point is appropriately located and if it and the target line are suitably free of visual detail, a flat form lacking connectedness and unity can be a more effective context than a coherent, connected, and unified three-dimensional form.

\section{DISCUSSION}

It seems unlikely that the OSE produced with Weisstein and Harris' type of forms can be accounted for exclusively by configurational variables. Fixationpoint location must be taken into consideration. The widely observed superiority of the three-dimensional coherent and connected object form was readily demonstrated in Experiment 2 with the standard fixation-point location, but a small shift in fixationpoint location eliminated the effect, and a flat line context, lacking in coherence and connectedness, was shown to be the more effective context. Movement of the fixation point does not reverse the very obvious differences in three-dimensionality, coherence, and connectedness between object and line contexts, but it does reverse the relative effectiveness of the two forms. What is responsible for the reversal of the OSE? Experiments 2 and 3 lend support to the view that the crucial factor is the amount of line detail on, or about, the fixation point. Movement of line detail toward the point of fixation in Experiment 2 impaired context effectiveness, and movement of line detail away from the point of fixation in Experiments 2 and 3 facilitated context effectiveness. In fact, when all line detail was moved a sufficient distance away from both the fixation point and the target line, the resulting flat, unconnected outline context proved to be the most effective context.

How general is the fixation location effect described? In the study of the OSE reported, the forms used were either identical to those used by Weisstein and Harris or were variants of those forms. Although these forms have been used very extensively in the literature (e.g., Klein, 1978; McClelland, 1978; Womersley, 1977), it must be conceded that they constitute a limited range of stimulus configurations, and it can be argued that this must constrain the generality of the findings. Although, as has been noted, an analysis on the object-line effect with a substantially broader set of forms has produced fixation location effects which closely parallel those reported (Earhard, 1980), a degree of caution is necessary. One question, in particular, to which the answer is not clear is whether these findings can be generalized to stimulus configurations that are very large and in which individual elements in the form are separated from one another by a sufficient distance to prevent lateral masking interactions (e.g., Banks \& Prinzmetal, 1976). The forms used in the research described are very small. All subtend a visual angle of less than $2 \mathrm{deg}$, and the potential for lateral masking interactions is large. The resolution of this question must await future research.

With respect to the question of why the amount of line detail at the fixation point should be important, our preference (which is elaborated more fully in Earhard, 1980) is to accept the arguments of Broadbent (1977) and Navon (1977) that perception involves both global and local processing operations. The global system is considered to have a shorter latency than the local system, and the low-level resolution of the visual terrain produced provides a foundation for the operation of the local analytic system that is responsible for more detailed, finegrain elaboration of the visual stimulus. The fixationlocation effect is judged to be a consequence of the way in which the local analytic system operates. Two assumptions are made: (1) that there are preferred ways to carry out the local analytic process, and (2) that the local analytic system begins automatically 
to process material at the point of fixation. The suggestion that there are preferred ways of carrying out the process of local analysis does not mean that a specific sequence or mode of analysis must be rigidly adhered to in processing a form, but only that general preferences may exist for various classes of form, or even for individual forms. These preferences will be determined in part by hardware-capacity constraints on the local system, that is, how much and how rapidly detail can be dealt with, and in part by the configurational character of the form presented, that is, how line detail is ordered and concentrated. This would mean that, in the case of tachistoscopic studies using line stimuli such as those reported, the fixation-point location will be extremely important. Contextual facilitation will depend to a large degree on whether the experimenter has positioned the fixation point at a location that coincides with the first step in the preferred processing sequence of a given form. In view of the large number of possible locations and the likelihood that processing preferences may vary with size (Kinchla \& Wolfe, 1979), it is rather improbable that this ideal state will always be achieved. If the fixation point falls on a nonpreferred location with a concentration of line detail, or there is a concentration of line detail between the fixation point and the to-be-specified target line, the results of Experiments 2 and 3 suggest that interference can be anticipated. The impairment can be reduced to some degree by shifting the fixation point, if possible, to a central location where there is little, or no, detail. This would minimize the amount of detail that would have to be dealt with at the initial point before pursuing an analytic sequence that adheres more closely to a preferred sequence. With respect to line detail on or about the target line, little can be done without altering the form. The data we have reported suggest that it can only serve to impair target-line detection.

Whether the interpretation of the fixation-location effect described is accepted or not, it seems to us that a more detailed analyis of the fixation-point and the way in which it interacts with figural infrastructure will prove rewarding. One interesting contrast already apparent is that between the emphasis placed on the importance of complex line intersection in computer recognition models and our finding that complex line intersections seem to be anything but ideal sites for fixation-point location in tachistoscopic studies.

\section{REFERENCE NOTE}

1. Williams, A., \& Weisstein, N. The time course of objectsuperiority. Unpublished manuscript, 1979.

\section{REFERENCES}

Banks, W. P., \& Prinzmetal, W. Configurational effects in visual information processing. Perception \& Psychophysics, 1976, 19, 361-367.

BarLow, H. B. Single units and sensation: A neuron doctrine for perceptual psychology? Perception, 1972, 1, 371-394.

Broadbent, D. E. The hidden preattentive processes. American Psychologist, 1977, 32, 109-118.

EARHARD, B. The line-in-object superiority effect in perception: It depends on where you fix your eyes and what is located at the point of fixation. Perception \& Psychophysics, 1980, 28, 9-18.

Hubel, D. H., \& Wiesel, T. N. Receptive fields, binocular interaction and functional architecture in the cat's visual cortex. Journal of Psychology, 1962, 160, 106-154.

HubEL, D. H., \& WiEsEL, T. N. Receptive fields and functional architecture in two non-striate visual areas (18 and 19) of the cat. Journal of Neurophysiology, 1965, 28, 229-289.

Kinchla, R. A., \& Wolfe, J. M. The order of visual processing: "Top-down," "bottom-up," or "middle-out." Perception \& Psychophysics, 1979, 25, 225-231.

KLEIN, R. Visual detection of line segments: Two exceptions to the object-superiority effect. Perception \& Psychophysics, 1978, 24, 237-242.

Lindsay, P. H., \& Norman, D. A. Human information processing: An introduction to psychology. New York: Academic Press, 1977.

MCClei.taAnd, J. L. Perception and masking of wholes and parts. Journal of Experimental Psychology: Human Perception \& Performance, 1978, 4, 210-223.

Milner, P. M. A model for shape recognition. Psychological Review, 1974, 81, 521-535.

Navon, D. Forest before trees: The precedence of global features in visual perception. Cognitive Psychology, 1977, 9, 353-383.

Pomerantz, J. R., Sager, L. C., \& Stoever, R. J. Perception of wholes and of their component parts: Some configural effects. Journal of Experimental Psychology: Human Perception \& Performance, 1977, 3, 422-435.

Prinzmetal, W., \& Banks, W. P. Good continuation affects visual perception. Perception \& Psychophysics, 1977, 21, 389-395.

Schendel, J. D., \& Shaw, P. A test of the generality of the word-context effect. Perception \& Psychophysics, 1976, 19, 383-393.

Sf KUlar, R. Spatial vision. In Annual review of psychology (Vol. 31). Palo Alto: Annual Reviews, 1974.

Selfridge, O. G. Pandemonium: A paradigm for learning. In The mechanization of thought processes. London: $\mathrm{H}$. M. Stationery Office, 1959.

Weisstein, N., \& Harris, C. S. Visual detection of line segments: An object-superiority effect. Science, 1974, 186, 752-755.

Weisstein, N., \& Maguire, W. Computing the next step: Psychophysical measures of representation and interpretation. In E. M. Riseman \& A. R. Hanson (Eds.), Computer visual systems. New York: Academic Press, 1980.

Williams, A., \& Weisstein, N. Line segments are perceived better in a coherent context than alone: An object-line effect in visual perception. Memory \& Cognition, 1978, 6, 85-90.

Womersley, M. A contextual effect in feature detection with application of signal detection methodology. Perception \& Psychophysics, 1977, 21, 88-92.

(Received for publication September 27, 1979; revision accepted July 24,1980 .) 